\title{
Supplementary document to:
}

"Super atmospheric water harvesting hydrogel with alginate chains modified with binary salts “

Akram Entezari, Mojtaba Ejeian, Ruzhu Wang ${ }^{1}$

${ }^{1}$ Corresponding author email address: $\underline{\text { rzwang@sjtu.edu.cn }}$ 


\section{S1-Experimental detail}

Chemicals:

The materials used in this study are listed in Table S1.

Table S1. Chemical characterization

\begin{tabular}{|l|l|l|}
\hline Chemicals & Features & Manufacturer \\
\hline $\begin{array}{l}\text { Carbon nanotube, multi- } \\
\text { walled, carboxylic acid } \\
\text { functionalized(FCNT) }\end{array}$ & $\begin{array}{l}\text { thin, extent of labeling: }>8 \% \\
\text { carboxylic acid } \\
\text { functionalized, avg. diam. } \times \mathrm{L} \\
9.5 \mathrm{~nm} \times 1.5 \mu \mathrm{m}\end{array}$ & Sigma-Aldrich, Inc. \\
\hline Sodium Alginate (SA) & $\begin{array}{l}\text { ACS reagent, } \geq 99 \%, 90 \%, \\
\mathrm{M} / \mathrm{G}=2: 1\end{array}$ & $\begin{array}{l}\text { Shanghai Macklin } \\
\text { Biochemical CO., Ltd. }\end{array}$ \\
\hline $\mathrm{LiCl}$ & ACS reagent, $\geq 99 \%$, & Aladdin \\
\hline $\mathrm{CaCl} 2$ & ACS reagent, $\geq 97 \%$, & Aladdin \\
\hline Deionized water (DI) & - & $\begin{array}{l}\text { Shanghai Macklin } \\
\text { Biochemical CO., Ltd. }\end{array}$ \\
\hline
\end{tabular}

Composite preparation:

First, we suspended 5\% wt. FCNT in water (mixed on a shaker for a long time and followed by 20 minutes ultrasonic). We then slowly added Sodium alginate to this suspension on a magnetic stirrer at $80^{\circ} \mathrm{C}$. We need a long time mixing to completely solve sodium alginate. Once alginate is completely dissolved in water, the following procedure was done for each of the composites:

- To make calcium alginates(C/FCNT): We dropped the as-prepared suspension into a saturated solution of calcium chloride. The process of gelation is very fast and sensible. After 30 hours of immersion, we filtered 
the particles of calcium alginate, and washed the particles with ethanol several times and dried them for 20 hours in $120^{\circ} \mathrm{C}$. In this process, calcium is expected to replace sodium in G-blocks of alginate chains.

- To make lithium alginate (L/FCNT): We dropped the as-prepared suspension into a lithium chloride saturated solution. The gelation process was very slow. After 30 hours, we filtered the particles of Lithium alginate with vacuum and washed the particles with ethanol several times. Due to its solubility issue, water or low purity ethanol cannot be used to wash this substance. After the washing process, we dried those for 20 hours at $120^{\circ} \mathrm{C}$. In this process, lithium is expected to replace sodium in alginate chains.

- To make binary alginate (Bina/ FCNT): we dropped the as-prepared suspension into a saturated calcium chloride solution. After 10 hours, the particles of calcium alginate were filtered and the resulting particles were soaked in lithium chloride saturated solution. Although lithium is not able to be replaced with calcium, the lithium chloride solution was used at a very small amount to prevent the diffusion of stored chloride calcium in the beads due to the concentration gradient of particles and external solutions. After 20 hours, beads were separated and washed several times with ethanol and dried for 20 hours at $120^{\circ} \mathrm{C}$. In this process, calcium is expected to replace 
Sodium from the G-blocks, and then sodium is replaced by lithium in the Mblocks.

Composites with layer structure

To test the surface temperature under sunlight, determination of thermal diffusivity and UV-vis-NIR test, we needed to have these materials in layer forms. To do this, instead of dropping the suspension in the corresponding salt, the solution is first injected into an as-fabricated cast and then placed in the solution of salt. We used two $5 \mathrm{~cm}$-in-5-cm glass slides to build the cast. We kept a certain distance between the glasses with a spacer $(5 \mathrm{~mm})$ and then wrapped it with multilayer paraffin. This wrapping process should not be so weak that the suspension discharged from the casts, and not so strong to hinder the penetration of salt inside.

\section{S2- Mechanism of sorption}

In most of studies Lithium and calcium salts were used as a component of sorbent [1-3]. Here, the replacement of sodium with these two salts aims to improve the structure shape and sorption properties. The proposed materials shows type II adsorption isotherm. This type of isotherm does not display any saturation point 
and in physical adsorption describes the adsorption of gas molecules to the macroporous and a multi-layer of adsorbed molecules is formed after the coverage of the adsorbent surface with mono-layer water vapor adsorbed molecules. But here chemical sorption occurs. Calcium Alginate is not soluble in water while lithium Alginate is soluble in water. Both of these substances are moisture adsorbent. The presence of unreacted salts in the composite body can be confirmed by FTIR examination. The presence of these hydrophilic salts can increase the adsorption capacity of these substances. The salts accumulated in this composite convert to hydrated salts and Lithium alginate slowly begins to dissolve in the presence of moisture. Eventually it becomes liquid over time. However, calcium alginate does not dissolve in absorbed moisture and remains solid. The adsorption capacity of calcium chloride is lower than that of the lithium chloride, but the disadvantage of dissolving lithium alginate limits its use. In the Bina composite, the calcium ion holds the polymer chains together from the G-block sites and strengthens the structure of the adsorbent, whereas $\mathrm{LiCl}$ and $\mathrm{CaCl}_{2}$ salt particles participate in adsorption function. The advantage of Bina is using both $M$ and $G$ blocks in the sorption. A combination of four mechanisms, adsorption by the stored salts, chemical reaction between vapor and two salts, enormous hydrogen bond formation between polymeric onion and water, and finally absorption by the 
formed salt aqueous solution, governs the water sorption behavior of the desiccant composites. Figure S1 shows the mechanism of sorption in salt crystals and alginate chain.

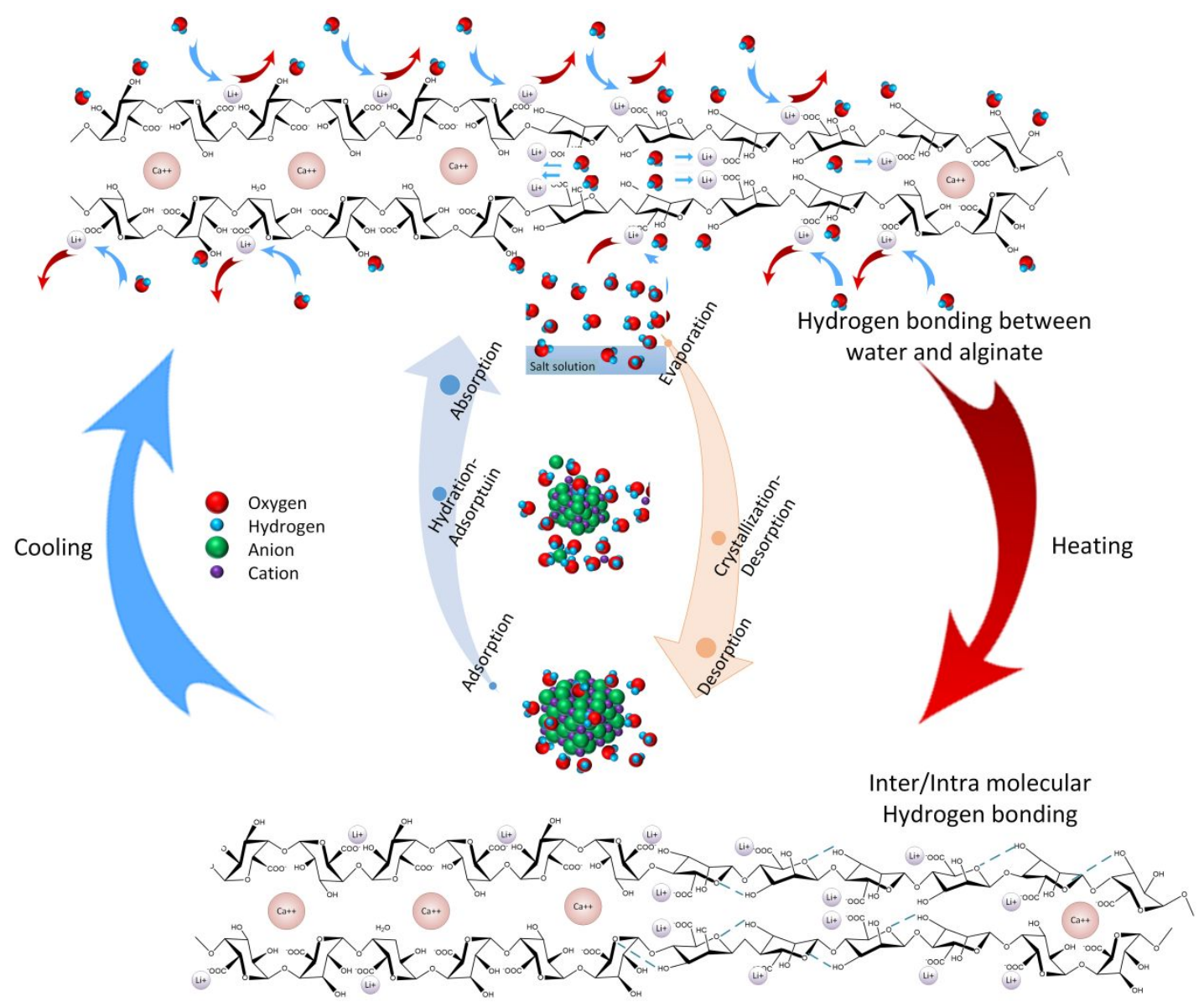

Figure S1. The mechanism of sorption in salt crystals and alginate chain: Possible hydrogen bonding occurred between water and alginate (surrounding) [4], and Salt crystals sorption mechanism (middle) 


\section{S3- FTIR analysis}

FT-IR spectroscopy was performed to investigate the physicochemical interactions between the salts, alginate, and FCNT (The used instrument: IR / Nicolet 6700, Thermo Fisher, USA). For this purpose, at first, the chemical bonds of the sodium alginate and FCNT were analyzed to better determine the bond changes after the mixture of the precursors. Figure S2 represents the FT-IR results of the raw materials.
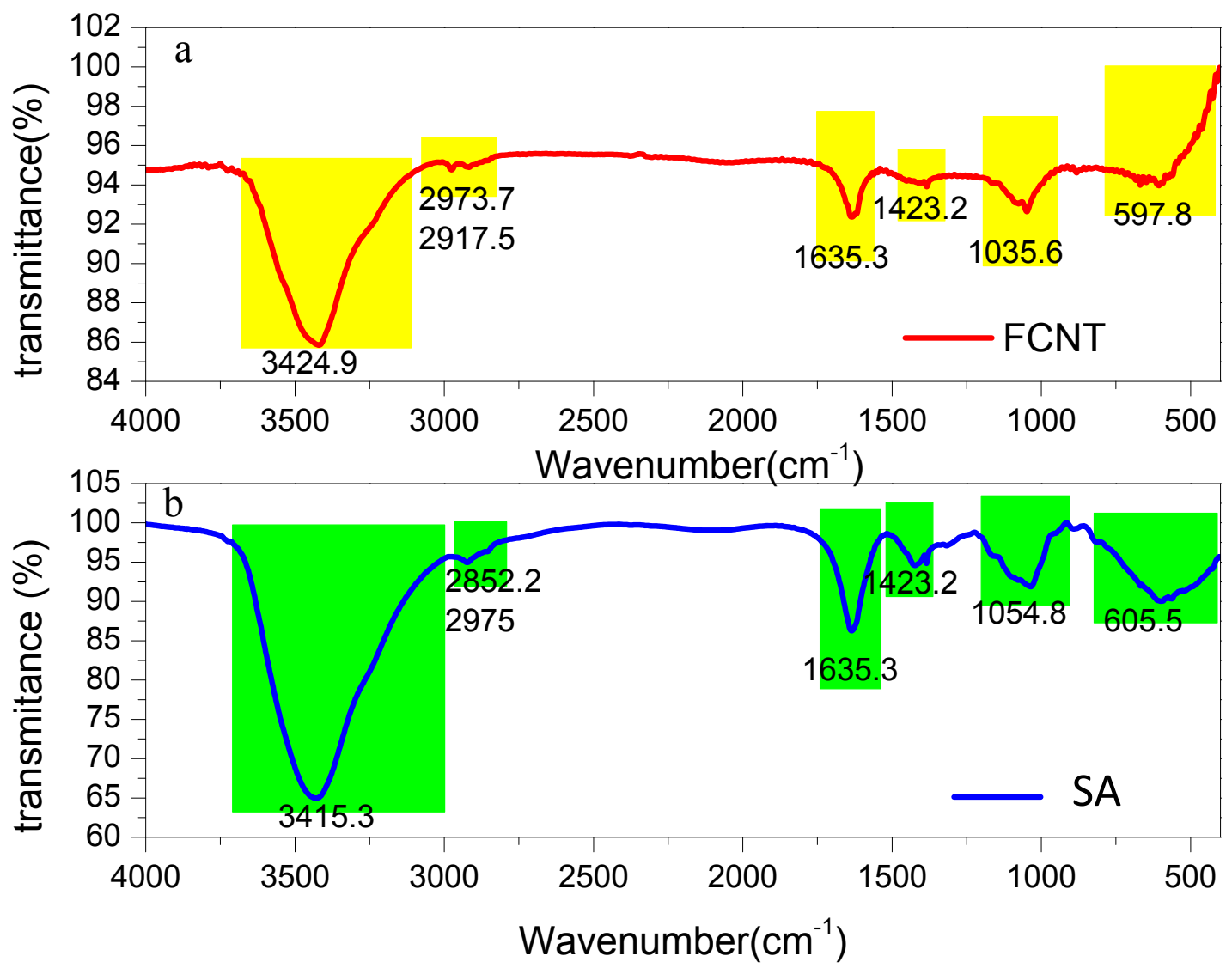

Figure S2. FT-IR spectra of (a) FCNT and (b) sodium alginate 
The FT-IR characteristic peaks of each component are reported in Table S2.

Table S2. The characteristic peaks of the used raw materials obtained from FT-IR analysis

\begin{tabular}{|c|c|c|c|c|}
\hline Component & Wavenumber $\left(\mathrm{cm}^{-1}\right)$ & Related vibration bond & $\begin{array}{c}\text { Transmittance } \\
(\%)\end{array}$ & Ref. \\
\hline \multirow[t]{7}{*}{ FCNT } & 597.8 & Aromatic rings & 93.9 & [5] \\
\hline & 1035.6 & $\begin{array}{l}\mathrm{u}(\mathrm{C}-\mathrm{O}-\mathrm{C}) / \\
\mathrm{u}(\mathrm{C}-\mathrm{O})\end{array}$ & 92.6 & [6] \\
\hline & 1423.2 & Sym. u( $\left.\mathrm{COOH}^{-}\right)$ & 94.0 & {$[6,7]$} \\
\hline & 1635.3 & $\begin{array}{c}\delta(\mathrm{O}-\mathrm{H}) / \\
\text { Asym. } \mathrm{u}\left(\mathrm{COOH}^{-}\right) / \mathrm{u}(\mathrm{C}-\mathrm{C} / \mathrm{C}=\mathrm{C})\end{array}$ & 92.3 & {$[5,7-9]$} \\
\hline & 2917.5 & Sym. u(C-H) & 96.4 & [10] \\
\hline & 2973.7 & Asym. u(C-H) & 94.8 & [10] \\
\hline & 3424.9 & $\mathrm{U}(\mathrm{O}-\mathrm{H})$ & 85.8 & [11] \\
\hline \multirow[t]{7}{*}{ SA } & 605.5 & Aromatic rings & 90.1 & [5] \\
\hline & 1054.8 & $\begin{array}{l}\mathrm{u}(\mathrm{C}-\mathrm{O}-\mathrm{C}) / \\
\mathrm{u}(\mathrm{C}-\mathrm{O})\end{array}$ & 91.9 & [6] \\
\hline & 1423.2 & Sym. u( $\left(\mathrm{OOHH}^{-}\right)$ & 94.6 & [12] \\
\hline & 1635.3 & $\begin{array}{c}\delta(\mathrm{O}-\mathrm{H}) / \\
\text { Asym. } \mathrm{u}\left(\mathrm{COOH}^{-}\right) / \mathrm{u}(\mathrm{C}-\mathrm{C} / \mathrm{C}=\mathrm{C})\end{array}$ & 86.3 & {$[5,7-9]$} \\
\hline & 2852.2 & Sym. u(C-H) & 94.9 & [10] \\
\hline & 2975 & Asym. u(C-H) & 95.0 & [10] \\
\hline & 3415.3 & $\mathrm{U}(\mathrm{O}-\mathrm{H})$ & 65.1 & [11] \\
\hline
\end{tabular}

U: stretching vibration

$\delta$ : bending vibration 
As can be seen in Figure S2 (a) and Table S2, the presence of symmetric and asymmetric $\mathrm{COOH}$ bonds at $1432.2 \mathrm{~cm}^{-1}$ and $1635.3 \mathrm{~cm}^{-1}$, respectively, in the FCNT spectrum confirms that the surface of the nanotubes was successfully modified by the carboxylic groups. In addition, the appearance of a broad and intense peak at $3415.3 \mathrm{~cm}^{-1}$, provides evidence of the presence of an alginic component with a high amount of hydroxyl groups in its structure. The FT-IR spectra of Lithium alginate (LA) and calcium alginate (CA) are shown in Figure S3.

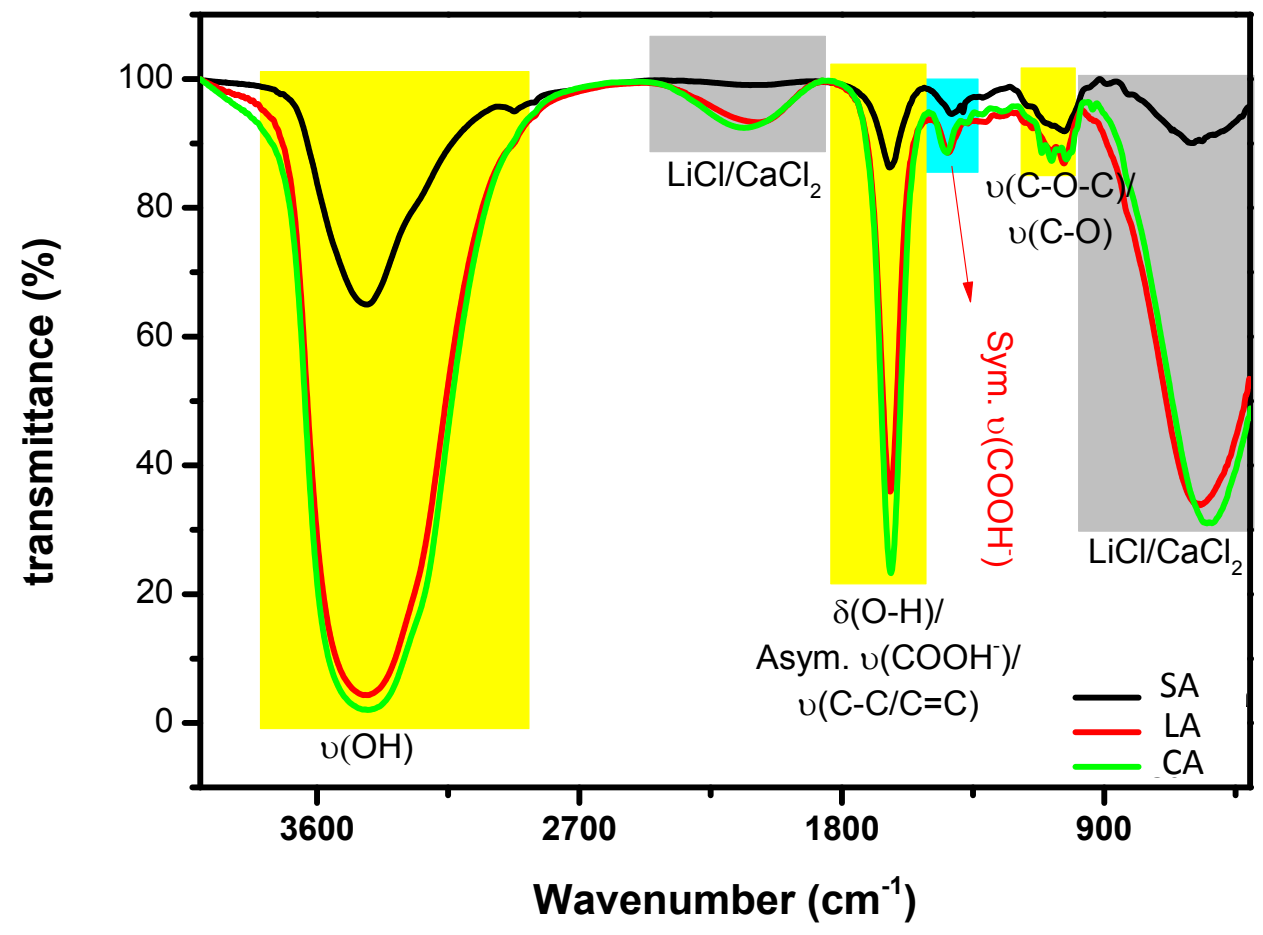

Figure S3. FT-IR spectra of sodium alginate, calcium alginate (CA) and lithium alginate (LA) 

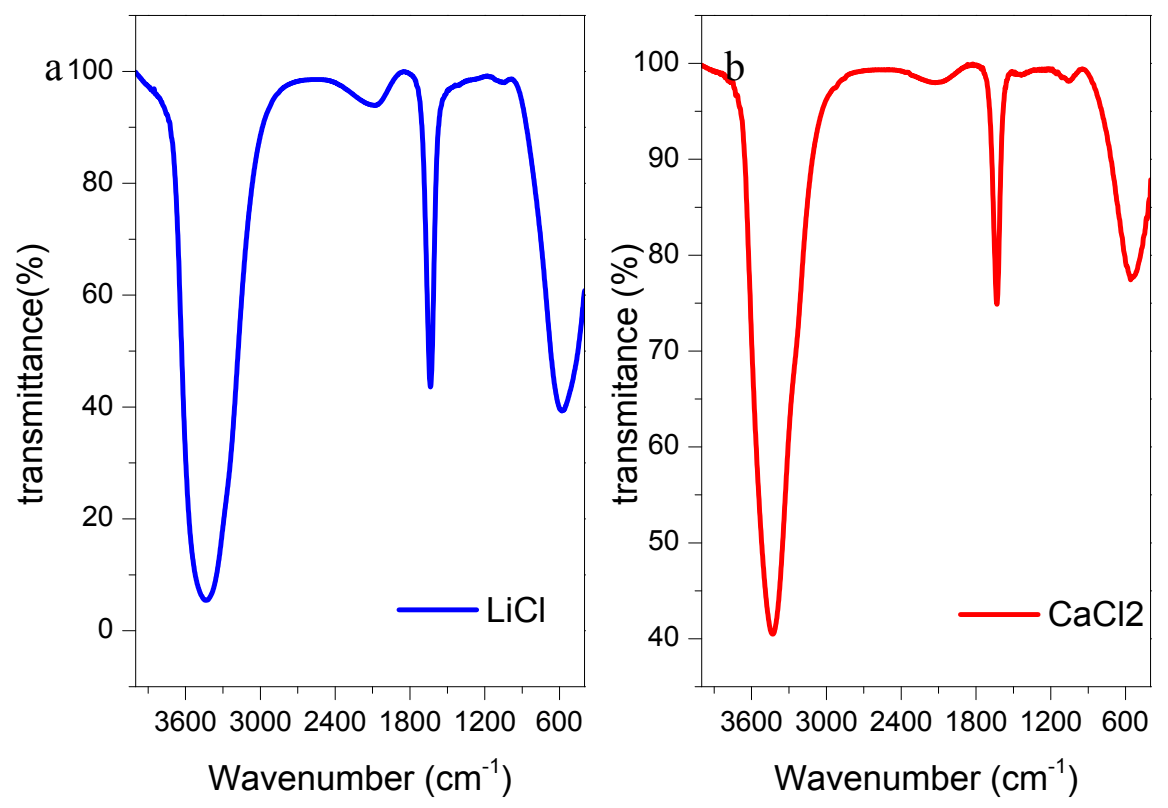

Figure S4. FT-IR spectra of (a) $\mathrm{LiCl}$ and (b) $\mathrm{CaCl}_{2}$ salts

As is shown in Figure $\mathrm{S} 3$, in addition to alginate peaks related to $-\mathrm{OH}, \mathrm{COOH}, \mathrm{C}-\mathrm{O}-$ C, and C-O bonds (Figure S2 (b)), two new peaks appeared at about $550 \mathrm{~cm}^{-1}$ and $2120 \mathrm{~cm}^{-1}$ attributed to the non-reacted chloride salts which remained in the system (see Figure S4) even after ethanol washing of the products. These remaining salts also affect the peak located at $1635 \mathrm{~cm}^{-1}$ and increase the intensity of it. The presence of these salts was seen in EDS and elemental mapping results too. Generally, by ignoring the effects of the remaining salts on the FT-IR results, the replacement of sodium ions with $\mathrm{Li}^{+}$and $\mathrm{Ca}^{2+}$ in the alginate structure should not change the spectrum of sodium alginate significantly due to lack of introducing any 
new chemical bonds in the system. However, the ion exchange process can show itself in the position of carboxylic ion bonds. In fact, due to differences in electronegativity of the $\mathrm{Na}^{+}, \mathrm{Li}^{+}$, and $\mathrm{Ca}^{2+}$, the attraction force between carboxylic anion and the used cations is different. The asymmetric stretching vibration of $\mathrm{COOH}$ anion bond located at about $1635 \mathrm{~cm}^{-1}$ cannot show the position shift due to overlapping with some other bonds vibration such as bending vibration of hydroxyl bonds and stretching vibration of benzene rings. The symmetric vibration of $\mathrm{COOH}^{-}$ bond at about $1320-1520 \mathrm{~cm}^{-1}$ of different alginates are shown in Figure S5.

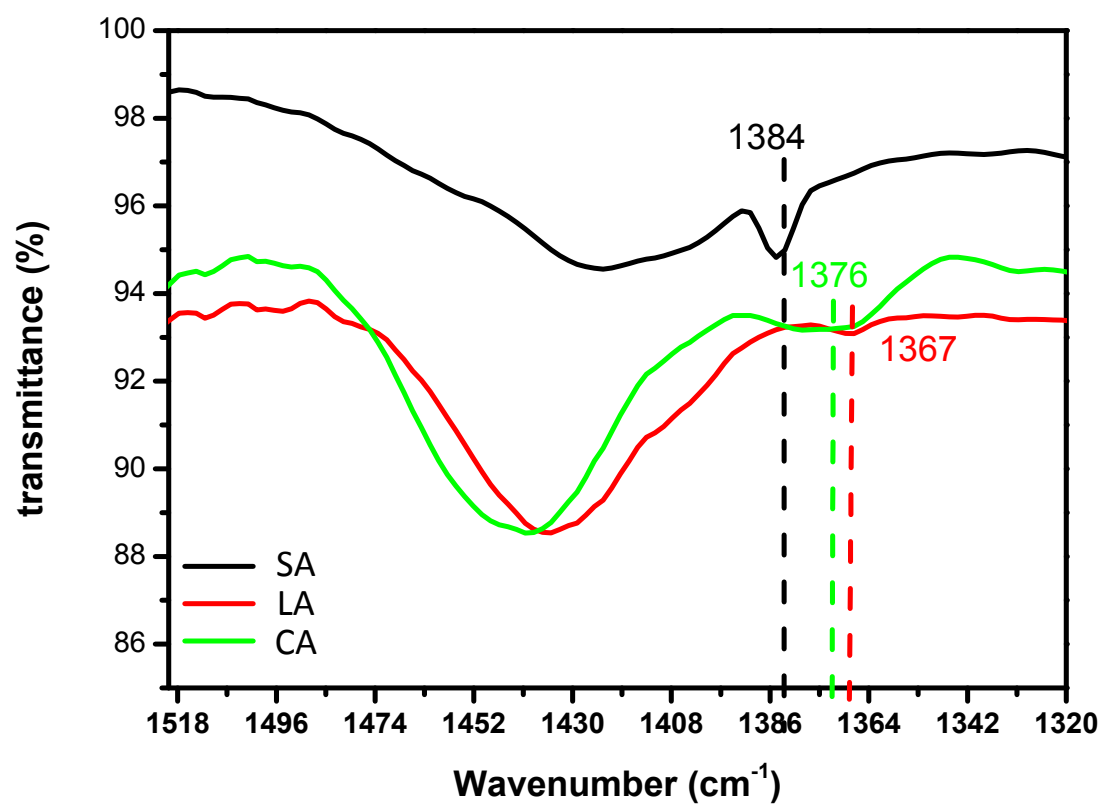

Figure S5. The symmetric stretching vibration of $\mathrm{COOH}^{-}$bond in FT-IR spectra of the studied alginates 
From Figure S5, a peak and a shoulder can be seen at $1420-1440 \mathrm{~cm}^{-1}$ and $1340-$ $1380 \mathrm{~cm}^{-1}$ which may be attributed to the non-extended carboxylic bonds and the extended $\mathrm{COOH}^{-}$bonds (due to electrostatically interaction with a cation), respectively. It is important to note that an extended bond needs lower energy to vibrate than a non-extended bond and so, the position of the extended bond is in lower wavenumbers compared to a bond without extension. Therefore, the redshift in the peak position of extended $\mathrm{COOH}^{-}$bond from $1384 \mathrm{~cm}^{-1}$ to $1367.8 \mathrm{~cm}^{-}$ ${ }^{1}$ and $1376 \mathrm{~cm}^{-1}$ for lithium alginate and calcium alginate, respectively, can provide evidence of successful ion-exchange after mixing the sodium alginate with $\mathrm{LiCl}$ and $\mathrm{CaCl}_{2}$ salts.

After producing the lithium alginate and calcium alginate by the ion exchange process, the effect of adding the $5 \%$ wt. FCNT nanoparticles into the products were investigated and the related FT-IR results are represented in Figure S6. 


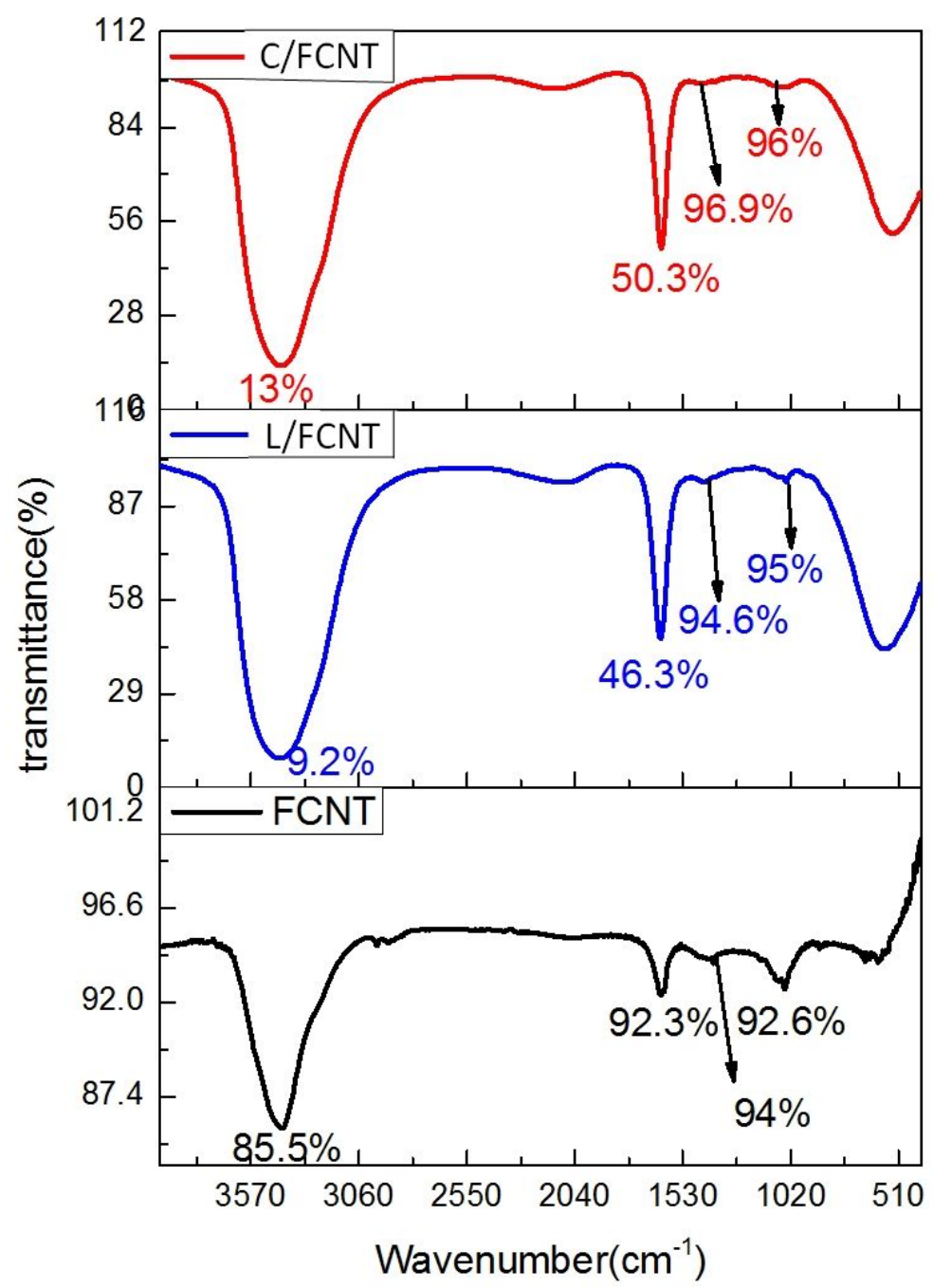

Figure S6. The FT-IR spectra of FCNT, lithium alginate-5\% wt. FCNT (L5), and calcium alginate$5 \%$ wt. FCNT (C5)

According to Figure S6, the higher intensity of the stretching and bending vibration of hydroxyl peaks located at about $3440 \mathrm{~cm}^{-1}$ and $1635 \mathrm{~cm}^{-1}$, respectively, compared to the peaks in the pure FCNT spectrum, indicating the presence of alginate components in the system. Despite the hydrogen-oxygen bonds, the intensity of carbon-oxygen bonds (such as $\mathrm{O}=\mathrm{C}-\mathrm{OH}, \mathrm{C}-\mathrm{OH}, \mathrm{C}-\mathrm{O}-\mathrm{C}$, and $\mathrm{C}-\mathrm{O}$ ) located at about $1000-1500 \mathrm{~cm}^{-1}$ were decreased compared to the related peaks in the pure 
FCNT spectrum, indicating that the later bonds may participate in the chemical bonding between the alginates and FCNT nanoparticles. A schematic of this chemical bonding was presented by Binata Joddar [13].

Figure S7 shows the effect of binary ion exchange $\left(\mathrm{Ca}^{2+}\right.$ and $\left.\mathrm{Li}^{+}\right)$on the FT-IR results of alginate components.

The more redshift of the extended $\mathrm{COOH}^{-}$peak position (from $1384 \mathrm{~cm}^{-1}$ to 1357 $\mathrm{cm}^{-1}$ ) in the binary cation exchange sample than the other components indicated that both lithium and calcium cations can cause more extension in the carboxylic anion bonds (see Figure S7 (a)). Furthermore, the decrement in the intensity of the non-extended carboxylic groups at about $1420-1440 \mathrm{~cm}^{-1}$ in the absence (Figure S7 (a)) and the presence (Figure S7(b)) of FCNT nanoparticles might be resulted that the amount of non-reacted anions in the alginate structures decreased after interaction between the components with both Li/Ca cations. 

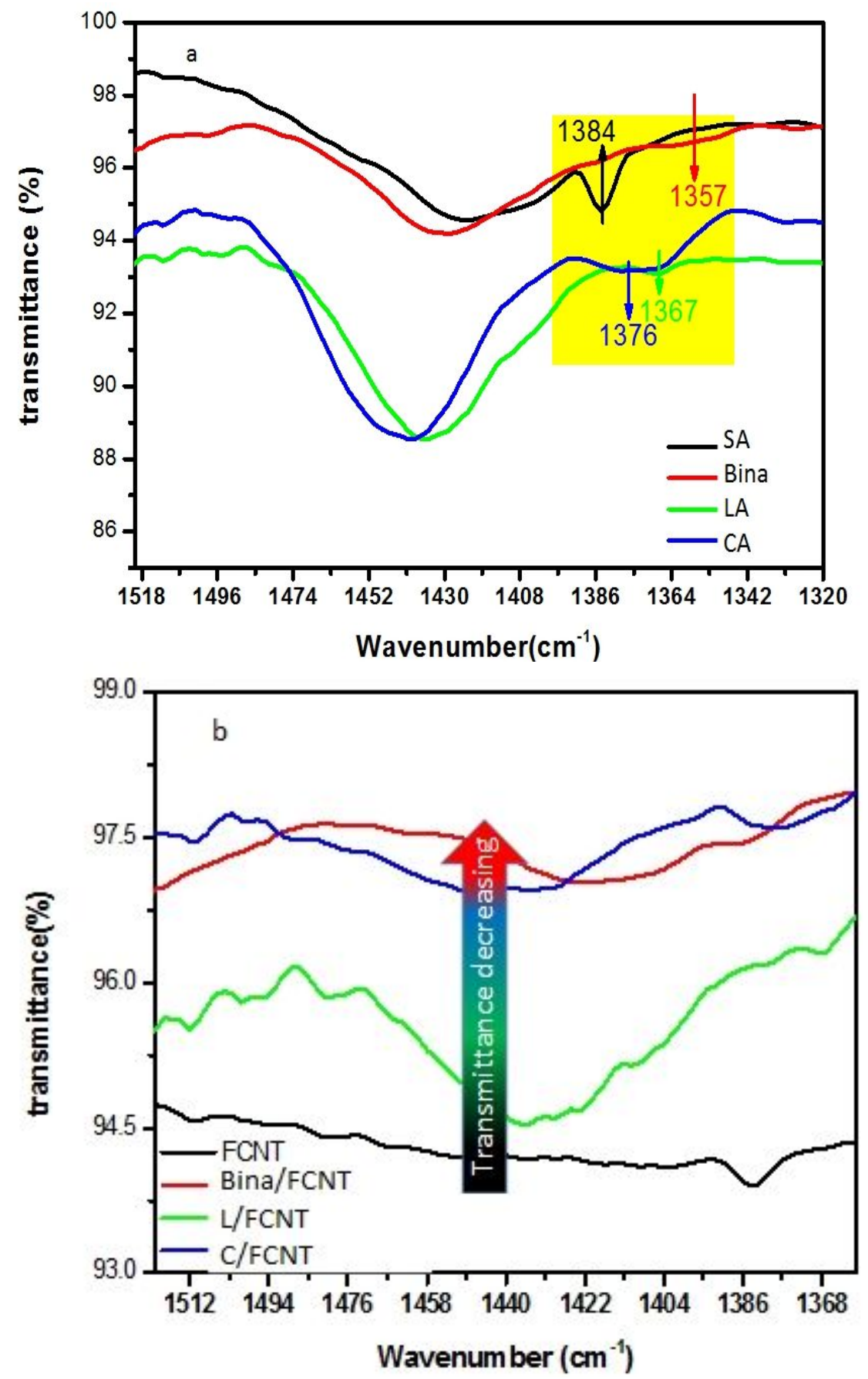

Figure S7. The symmetric stretching vibration of $\mathrm{COOH}^{-}$bond in FT-IR spectra of the (a) sodium, calcium, lithium, and binary alginates and (b) pure FCNT, lithium alginate-5\% wt. FCNT (L/FCNT), calcium alginate- $5 \%$ wt. FCNT (C/FCNT), and binary alginate- $5 \%$ wt. FCNT (bina/FCNT) 


\section{S4- SEM and EDS}

The composites' morphology was characterized using Scanning Electron Microscopy (SEM) micrograph, and the EDS analysis was applied to detect-measure elements in or on the surface of the samples.

The samples were placed on a brass hold and a thin coat of gold under vacuum was deposited on them, C/FCNT morphology and the surface structure were examined with TESCAN-MAIA/ WITEC/MAIA3 GMU model 2016 (see Table S3). The viewed structure was slightly similar to Li et al observation[14] but the fiber-like part of the matrix was not observed in our experiments.

Table S3. SEM and EDS analysis for C/FCNT

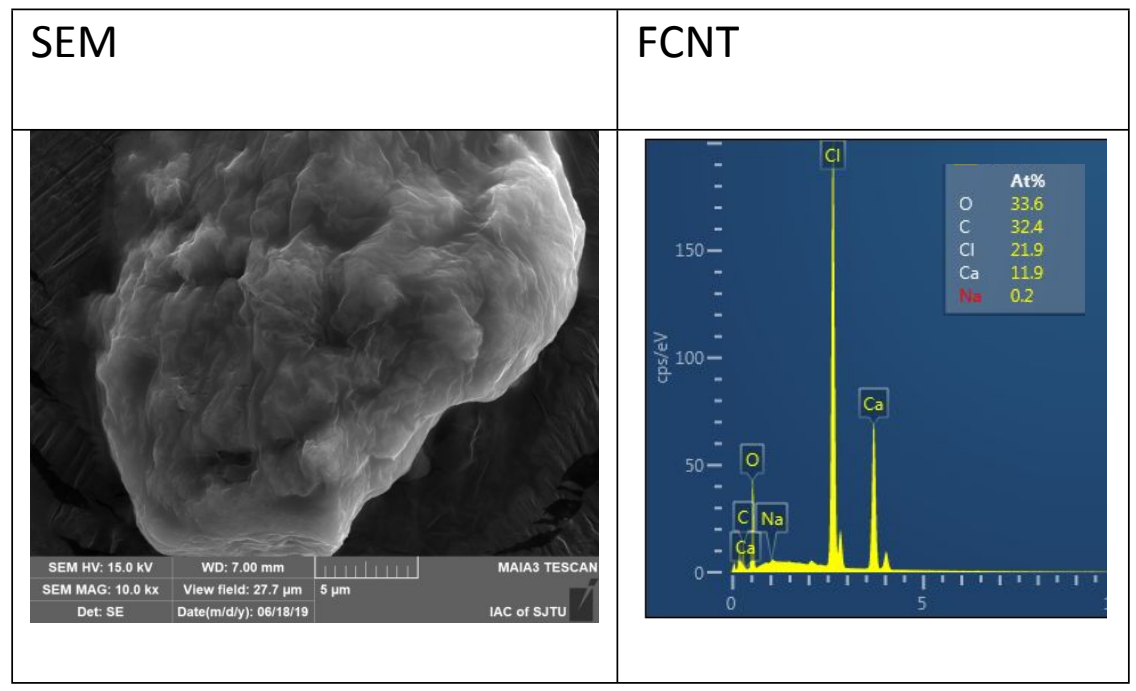


As far as this instrument cannot detect Li element, *GAIA3 GMU Model 2016/*GAIA3 was used for L/FCNT and Bina/FCNT. TOF-SIMS provides detailed elemental information about the surface and thin layers. Table S4 depicts the L/FCNT analysis. In SEM photos several micro-pores can be seen. Furthermore, despite C/FCNT, the surface is covered by small cracks. The top view and depth map confirm the presence of $\mathrm{Li}$ in the sample almost uniformly.

Table S4. SEM and TOF-SIMS analysis of L/FCNT

\begin{tabular}{|c|c|c|c|}
\hline SEM & Top view of Li map & Li map in-depth \\
\hline & & \\
\hline
\end{tabular}

Table S5 demonstrates the morphology of Bina/FCNT, as it can be seen, the surface has some cracks like L/FCNT. The TOF-SIMS analysis result shows the presence of $\mathrm{Li}$ and $\mathrm{Ca}$ in the superficial layer and also in the thin layer of this material. The quantity of these two elements is almost in a constant levels indepth direction. 


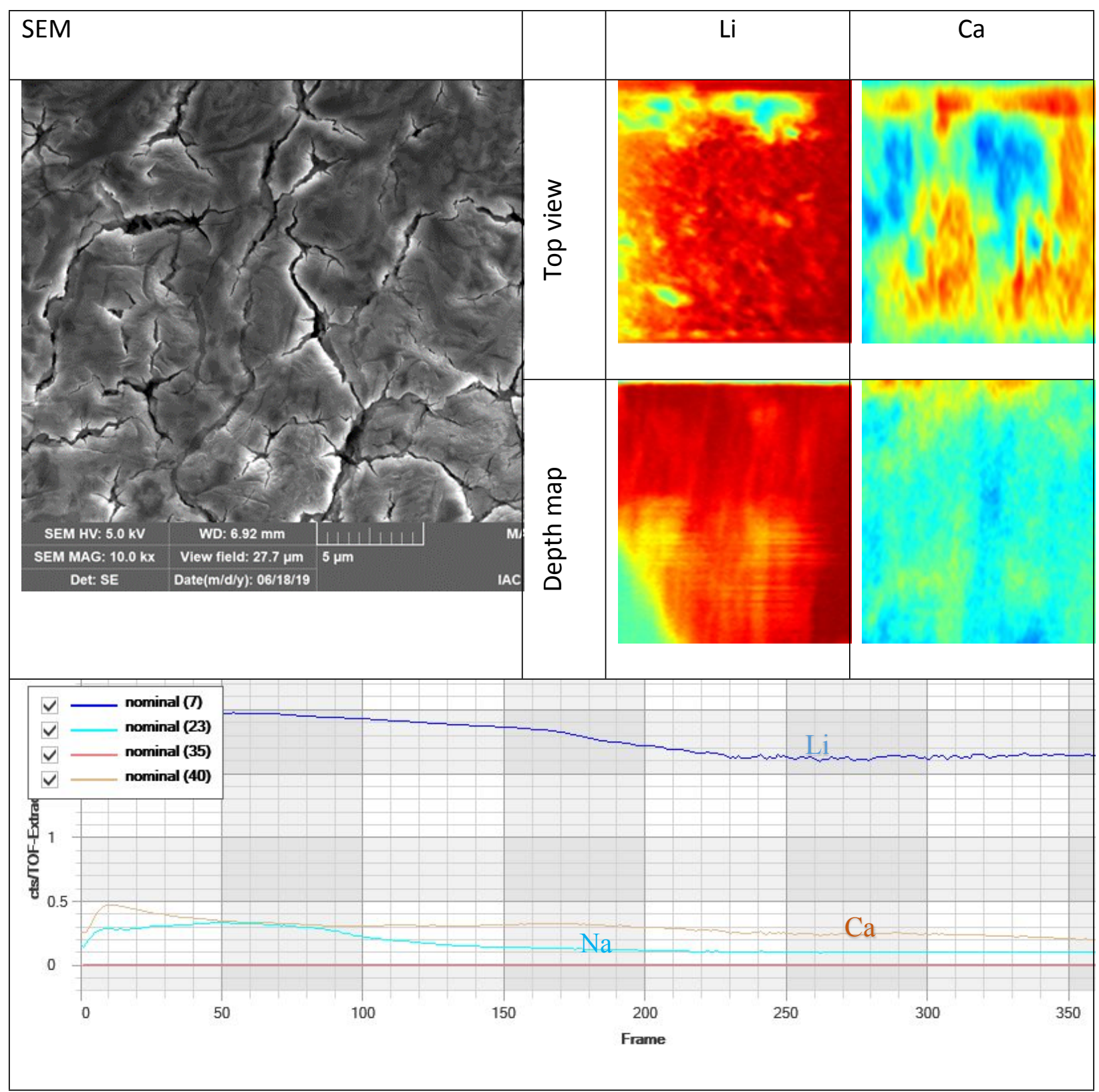


S5- Thermal distribution on the surface

The temperature of surface of the material under one sun radiation (created by a solar simulator EOSUN (Xee150 Xenon Lamp Power Supply) ) is investigated by a Thermal Imaging Camera (FLIR T620 HD) shown in Figure S8.
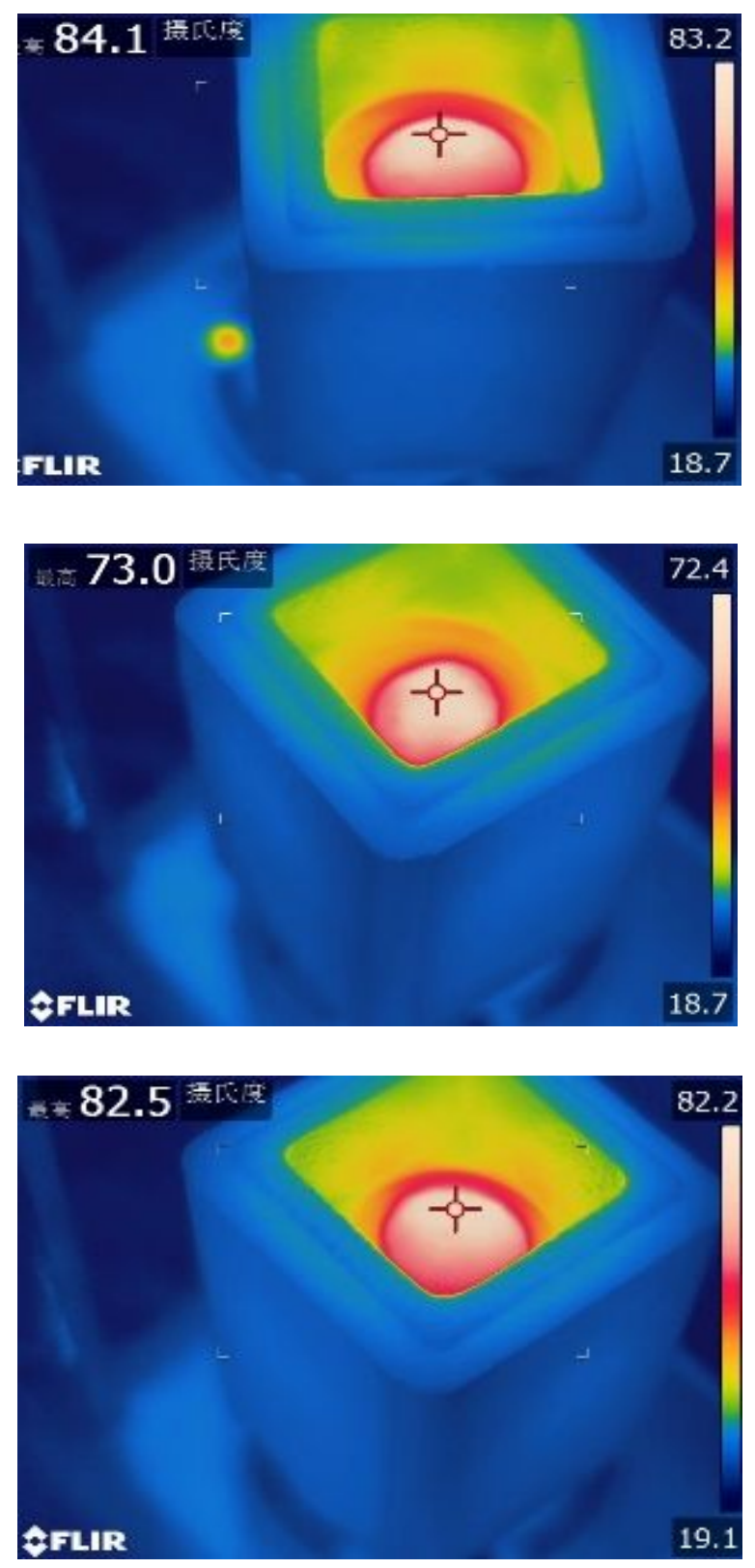

Figure S8. The surface temperature of C/FCNT (top), L/FCNT (middle) and Bina/FCNT(bottom) under one sun irradiation. 


\section{S6-Energy balance in the materials beads}

The heat loss analysis from the sorbent to open environment are shown vide infra.

In this case the net energy absorbed from the sun must equal the long-wavelength radiation exchange with the surroundings, convection transfers to the surroundings, heat for evaporation and finally conduction $\left(\mathrm{Q}_{\mathrm{cond}}\right)$ to the lower layers or[15]

$\left(\frac{\mathrm{q}}{\mathrm{A}}\right)_{\text {sun }} \alpha_{\text {sun }}=\alpha_{\text {low temp }} \sigma\left(\mathrm{T}_{\text {surface }}^{4}-\mathrm{T}_{\infty}^{4}\right)+\mathrm{h}\left(\mathrm{T}_{\text {surface }}-\mathrm{T}_{\infty}\right)+\hat{\mathrm{m}} \mathrm{h}_{\text {evap }}+\mathrm{Q}_{\text {cond }}$

where $\alpha_{\text {sun }}, \alpha_{\text {low temp }}, \sigma, \mathrm{T}_{\text {surface }}, \mathrm{T}_{\infty}, \mathrm{h}, \mathrm{R}_{\text {sun }}, \hat{\mathrm{m}}$ and $\mathrm{h}_{\text {evap }}$ are the absorptivity for solar radiation, the absorptivity for low-temperature radiation, Stefan-Boltzmann constant, the surface temperature, the surroundings temperature, the convection heat transfer coefficient, the evaporation mass flux and Enthalpy of vaporization, respectively. The absorptivity test shows that $\alpha_{\text {sun }}$ and $\alpha_{\text {low temp }}$ for this black material can be considered equal $97 \%$. $\mathrm{T}_{\text {surface }}$ and $\mathrm{T}_{\infty}$ are the almost $70^{\circ} \mathrm{C}$ (experiments under one sun raddiation) and $25^{\circ} \mathrm{C}$, respectively. Also, $\mathrm{h}$ can be considered $5 \mathrm{~W} / \mathrm{m}^{2} / \mathrm{K}[16]$.

$$
\begin{aligned}
& 1000 \times 0.97=0.97 \times 5.669 \times 10^{-8}\left(343^{4}-298^{4}\right)+5 \times(343-298)+\hat{\mathrm{m} h} \text { evap }+1(2 \\
& \hat{\mathrm{m} h} \text { evap }+\mathrm{Q}_{\text {cond }}=417.5 \mathrm{~W} / \mathrm{mm}^{2}
\end{aligned}
$$


The total energy loss from system are: reflection energy loss (3\%) and heat loss by convection (22.5\%) and radiation (32.7\%). The total energy remaining for water evaporation and conduction on the surface is calculated to be $41.8 \%$.

If we consider the energy balance boundaries around the material, conduction is not a loss from system. However, The low thermal conductivity causes to high thermal gradient on the surface. So the evaporation rate on the surface increases and consequently, the water concentration on the surface decreases. This leads to higher mass transfer from the core to the surface by diffusion.

Figure S9 shows the energy and mass balance in a material bead.

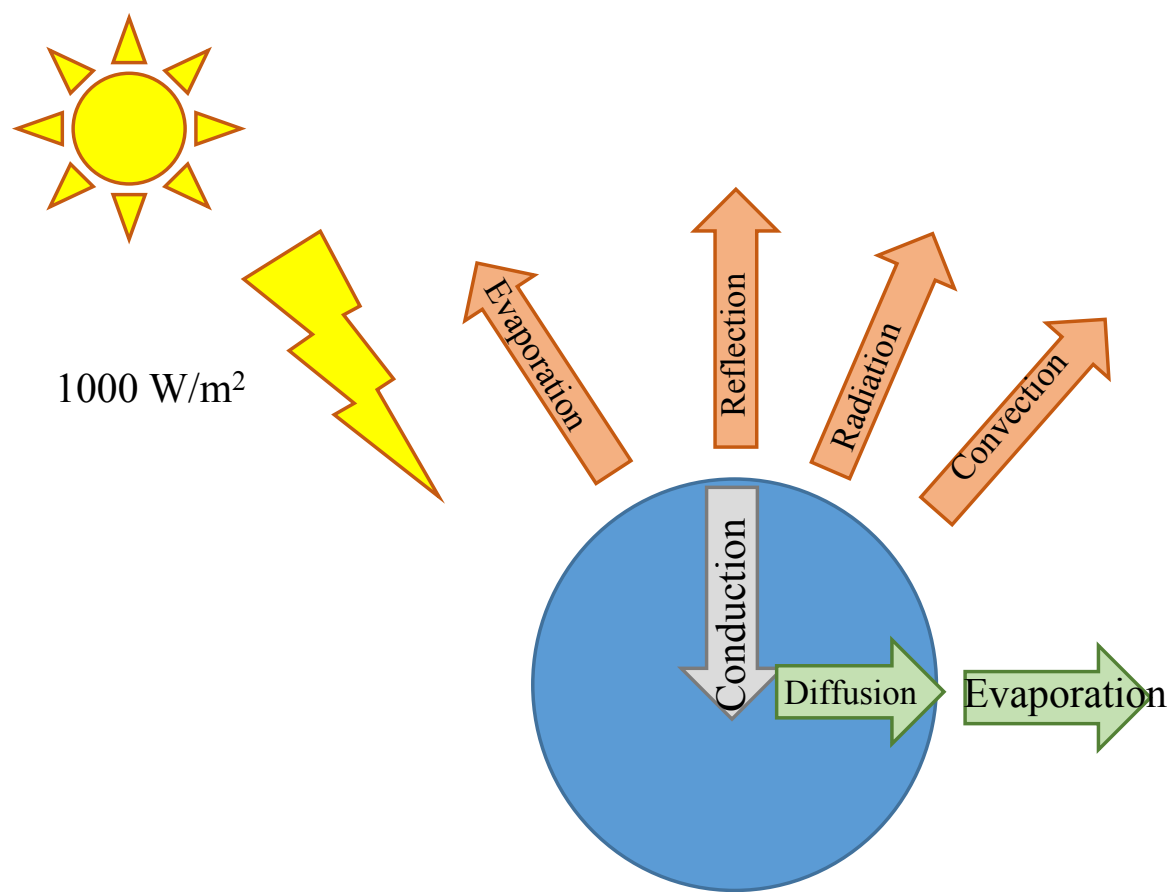

Figure S9. The energy an mass balance on the material surface. 
S7- The optimized bed configuration and cycle time

Delinquency is better to be avoided but Bina is strongly hygroscope and the delinquency time needs to be determined. Thus, some experiments were carried out to see the delinquency phenomenon. Two plates of material were used, one containing mono layer scattered beads, and the other one containing multi layers of material beads. The plates were placed in different relative humidity and were observed to see when the leakage happens. The leakage time and their weight were recorded. In $20 \%$ of $\mathrm{RH}$, no leakage was observed, so to depict the time in Figure S10, the inverse value of leakage time were used. The area of each circle represents the weight of material when the leakage starts (These numbers are written on the circles). As can be seen, the mono layer structure can adsorb more vapor and the leakage happens much faster in them. It can be related to this fact that in multi layers, the lower layers are not exposed to air directly and the upper layer's adsorbed water pass trough the beads and is collected on the lower layer and they can not contribute in adsorption. These experiments show that this sorbent is better to be used in one layer in the structure like a thin coating on a surface. To avoid the leakage we can also design cycles in very short humidification time (46, 32 and 19 min for $40 \%, 60 \%$ and $80 \%$, respectively), followed by the desorption process. 


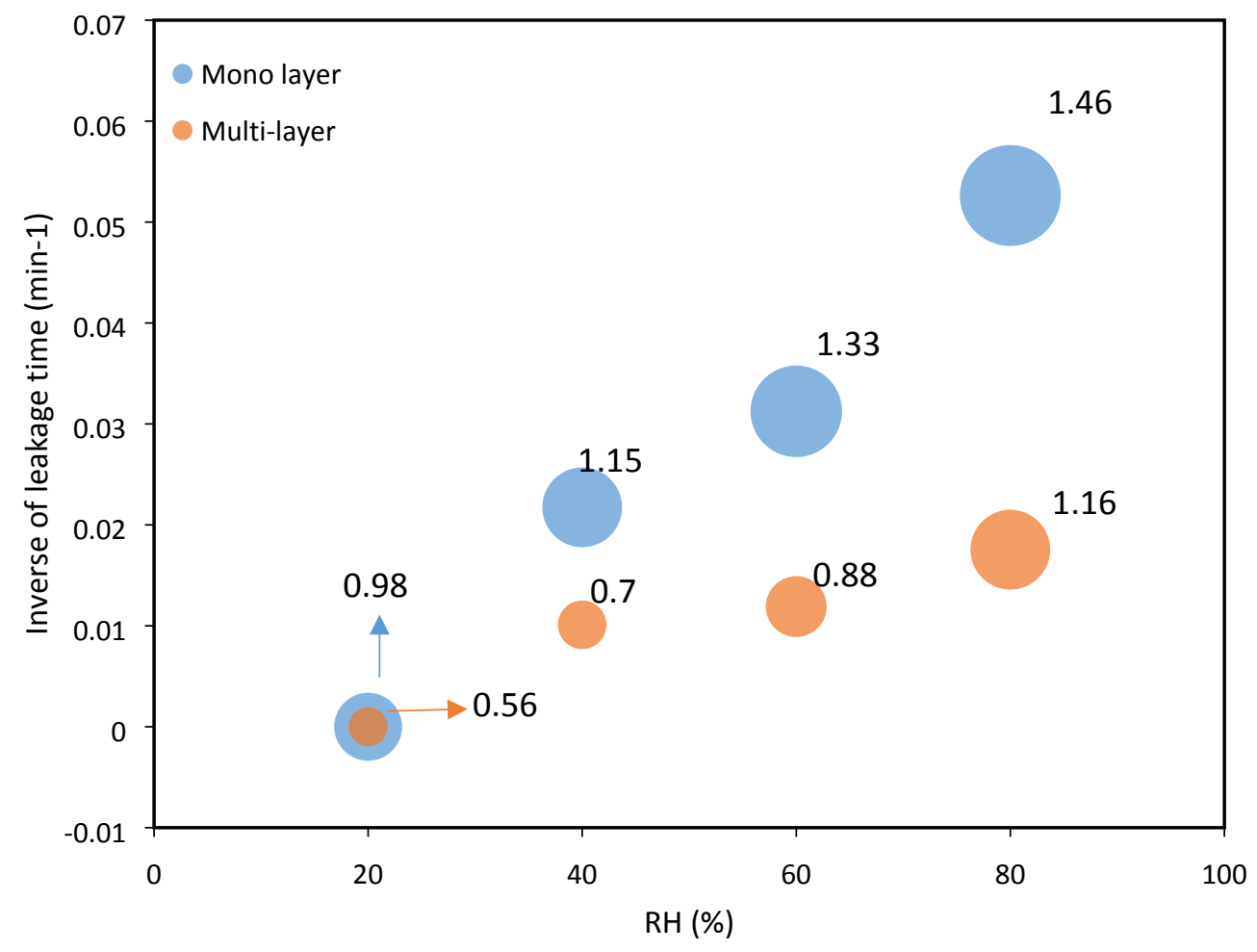

Figure S10. The inverse of Leakage time ( $y$-axis) and delinquency weigh ( the circles area) in different configuration of bed.

The real system design could be considered in two ways to avoid delinquency: 1 . Using the kinetic data of delinquency to design its cycle time, thus one day with sun radiation could yield many cycles for adsorption and desorption, $5 \sim 10$ repeated cycles could be possible according to the measured data, thus the AWH system could be very efficient as the uptake of water could be thereby increased significantly. 2. Using a porous matrix like activated carbon fiber to support Bina/FCNT, any possible delinquency liquid could be sustained in the sorbent complex. However the operation cycle time is still needed to be optimized to keep Bina/FCNT for long term stable. 


\section{S8-Literature review}

Table S6 summarized the adsorbents which are studied in recent years for AWH applications. Although in area with high humidity, where vapor compression system is more efficient, in $\mathrm{RH}<60 \%$, it is not practical. Some MOFs are good especially at low $\mathrm{RH}$, such as $\mathrm{CoCl}_{2}$ (BTDD, now this Bina/FCNT is much better than MOF even at low $\mathrm{RH}$, for example at $\mathrm{RH}=20 \%$ Bina/FCNT can adsorb $1.4 \mathrm{~g} / \mathrm{g}$ while at $\mathrm{RH}=30 \%, \mathrm{CoCl}_{2}$ (BTDD) adsorb almost $0.9 \mathrm{~g} / \mathrm{g}$. 
Table S6. Summarized of several promising materials that can be applied in water harvesting beds

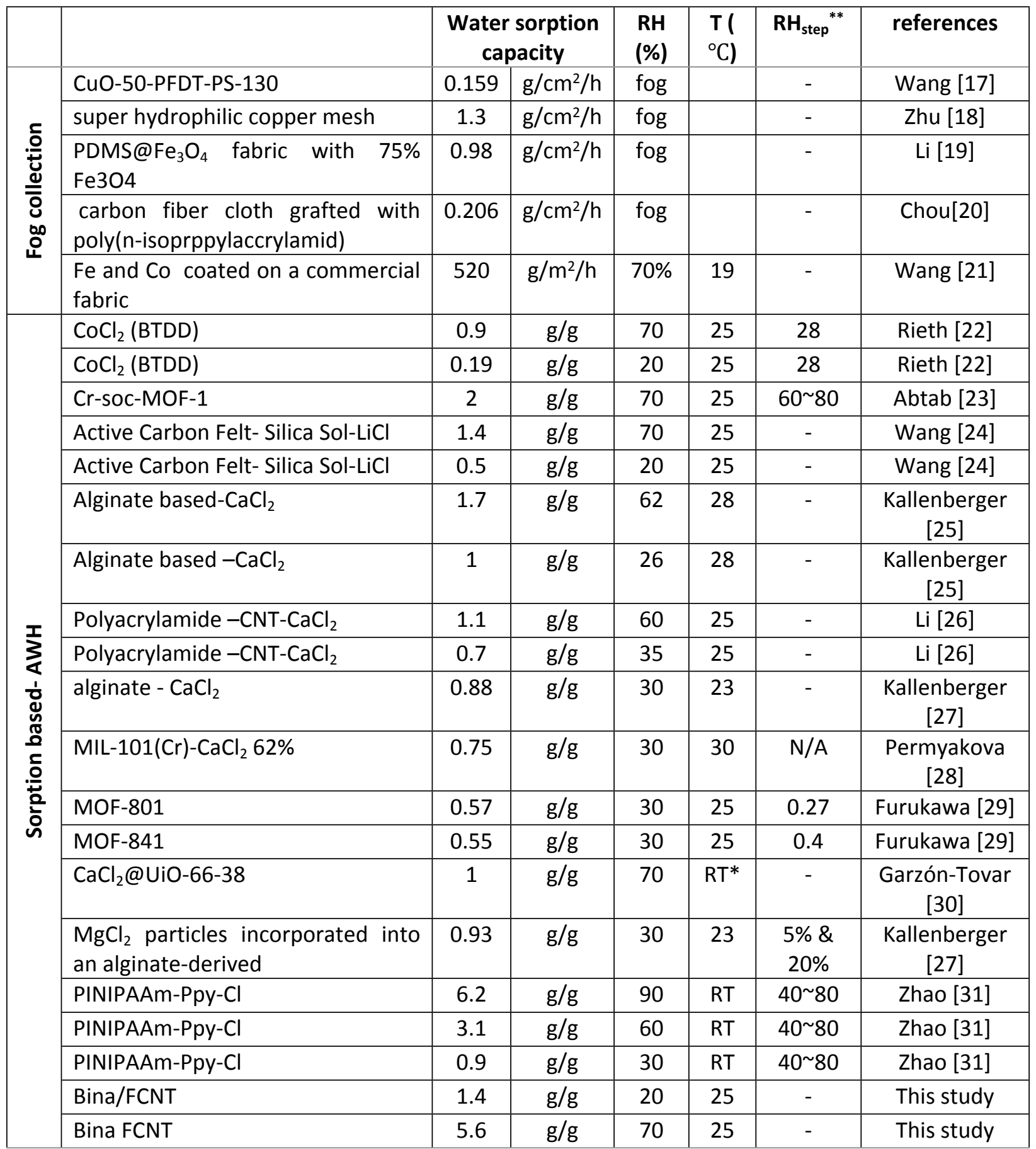

*: RT: Room temperature

$* *: \mathrm{RH}_{\text {step }}$ :The relative humidity that one sudden step happens. High adsorption capacity in low $\mathrm{RH}$, makes adsorption more reasonable at low RHs, compared to the electrical cooling. 
S9- The prototype design

The detail of the design and optical photo of the prototype, while running, is presented here (Figure S11). In the condensation process, after the formation of drops, the distilled water slips on the glass cover by gravity, and pours into the side channel embedded in the device. The 35 -degree slope of the glass cover prevents the drop from being heavy enough to break directly into the adsorbent chamber.

The side channel also has a slight slope that drives water droplets
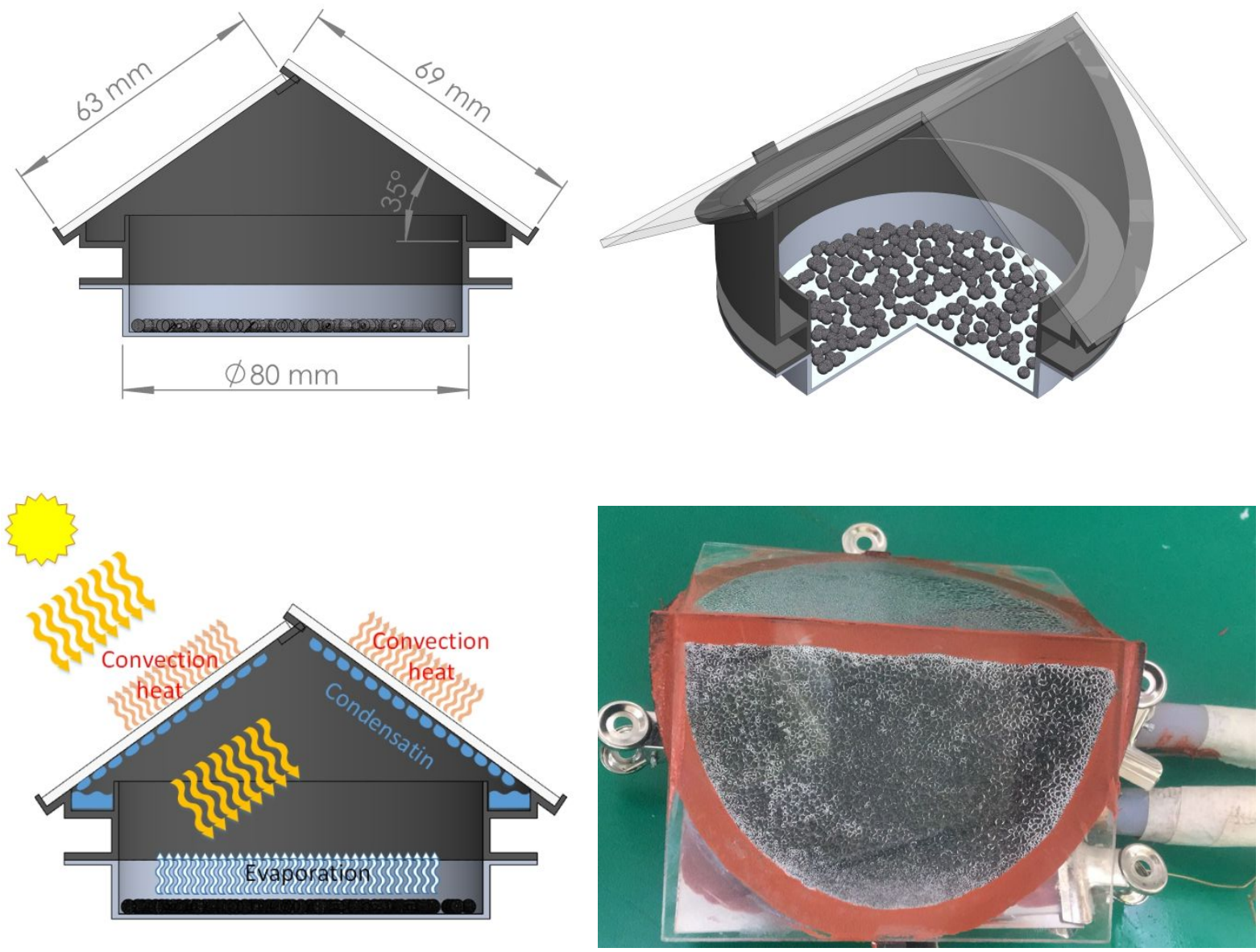

Figure S11. The schematic of device with details $(a, b)$ and the operation concept (c) and optical picture of the prototype (d). 


\section{S10- The performance under natural sunlight}

In this study, to assess different desorption temperature, we used thermo-bath. Since the experiment with water bath does not rigorously demonstrate the novelty of combining both the light absorption and water adsorption together in a single device, on $11^{\text {th }}$ of November 2019, a set of experiment was done on the roof of laboratory, located in Shanghai Jiao Tong University. To do this experiment, the bottom of device was isolated to avoid heat loss through it. The bed contains several layers of sorbent grains $(5.771 \mathrm{~g})$, which was placed in chamber for 6 hours in $\mathrm{RH}=30 \%$ and $\mathrm{T}=20^{\circ} \mathrm{C}$. It could sorb $8.948 \mathrm{~g}$ water, and the sample was placed on the roof at 9 am and the photos were taken during desorption process (Figure S12 $a, b)$. The temperature of glass and the bed was recorded and depicted in Figure S11c. Figure S12d shows the Solar radiation intensity and the ambient temperature. The maximum recorded temperature of bed was $66^{\circ} \mathrm{C}$ and the maximum solar radiation intensity was $660 \mathrm{~W} / \mathrm{m}^{2}$. The maximum temperature of glass was $39^{\circ} \mathrm{C}$ which is higher than the measured corresponding value in the tests with water bath. The reason can be contributed to the light sorption of glass and the condensed droplets. Since the solar radiation was not strong enough, the experiment sopped ay 2:30 and the yield was almost $3.376 \mathrm{ml}$ water . (We kept 
recording the temperature of bed and glass of prototype and reported them in

Figure S12c).

a

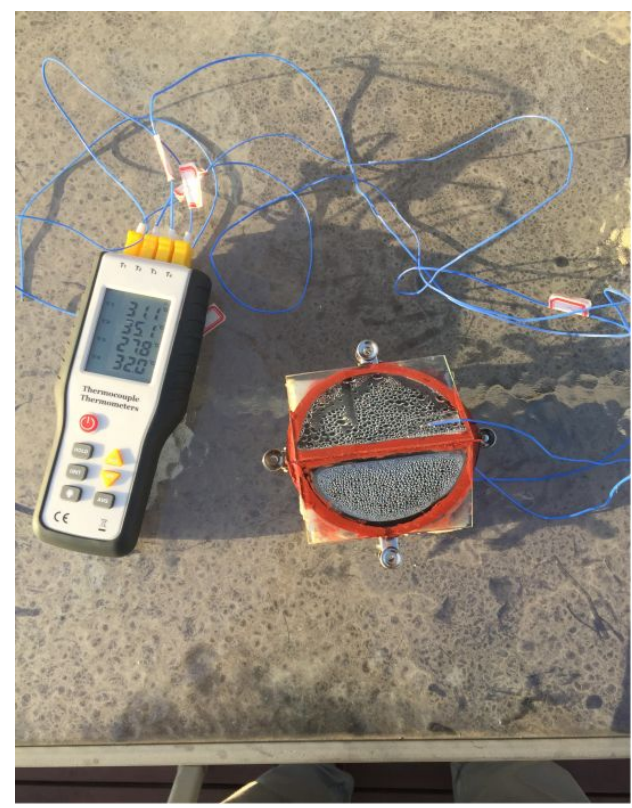

$\mathrm{C}$

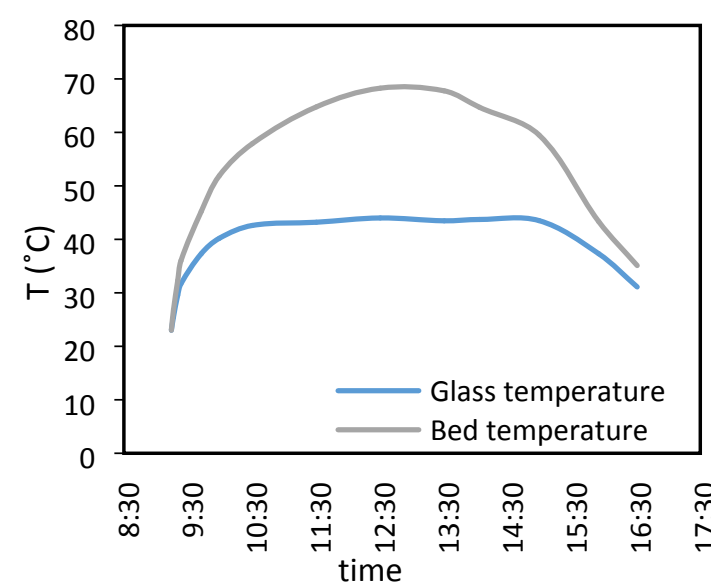

b

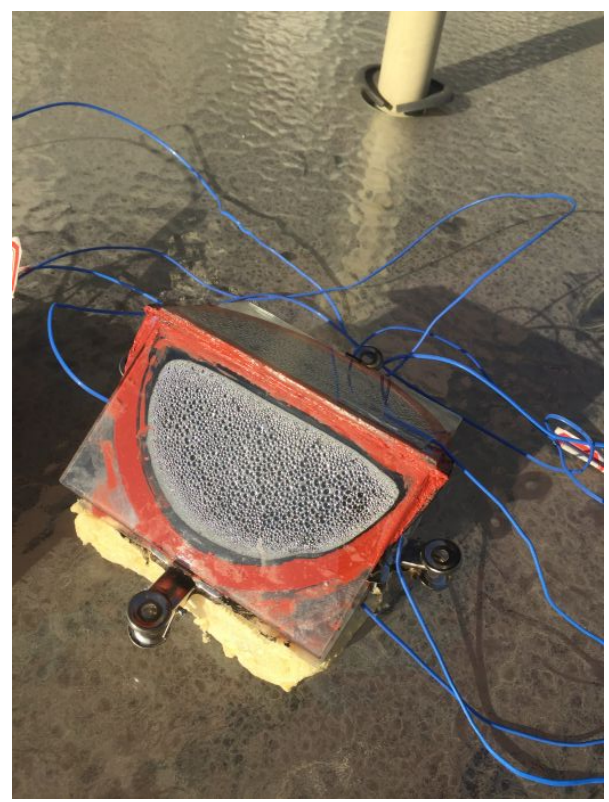

d

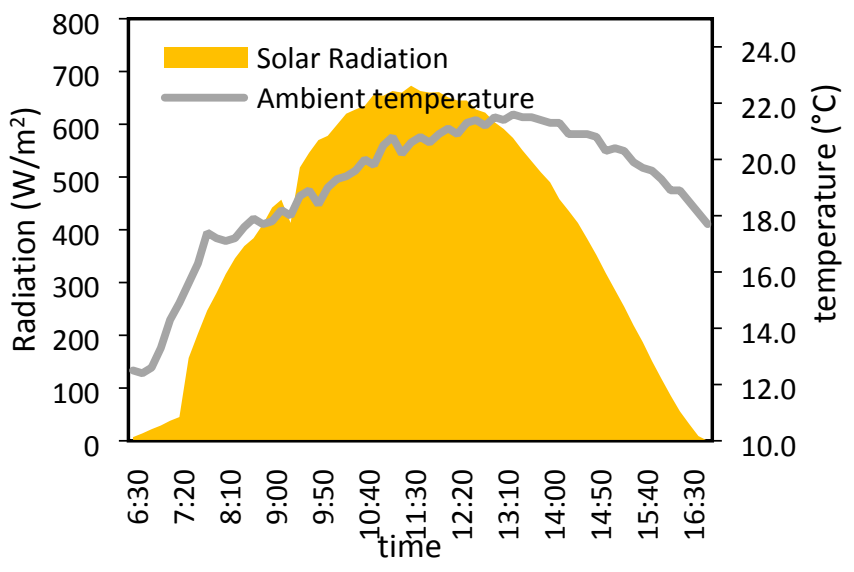

Figure S12. The performance of prototype under natural sunlight. a,b. The optical pictures of

prototype. c. The glass and the bed temperature profile and $\mathrm{d}$. The solar radiation intensity and ambient temperature. 


\section{S11- Prototype Energy consumption}

The heat energy entered into the system is divided into two parts. The first part is about providing desorption heat to the adsorbent particles. The total desorption heat dissipated in the prototype is calculated from formula (4)

$$
\mathrm{E}_{\text {des }}=\mathrm{m}_{\text {adsorbate }} * \mathrm{~h}_{\mathrm{des}}
$$

Where, $\mathrm{E}_{\mathrm{des}}$ is the total desorption heat, $\mathrm{m}_{\text {adsorbate }}$ is mass of desorbed adsorbate and $\mathrm{h}_{\text {des }}$ is desorption heat per unit of mass. The desorption heat is obtained based on TGA data of $2768 \mathrm{~J} / \mathrm{g}$.

Part of the heat energy transferred into the system is transmitted to the glass coating by convection. To calculate the heat transfer coefficient between the substrate and the glass, we used the Zheng et al. method presented for Solar Stills [32].

$$
\begin{aligned}
& \mathrm{h}_{\mathrm{c}}=0.2\left(\mathrm{R}_{\mathrm{ac}}\right)^{0.26} \frac{\mathrm{k}_{\mathrm{f}}}{\mathrm{d}} \\
& \mathrm{R}_{\mathrm{ac}}=\frac{\mathbb{d}^{3} \rho \mathrm{g} \beta}{\mu \alpha} \Delta \mathrm{T}^{\prime} \\
& \Delta \mathrm{T}^{\prime}=\left(\mathrm{T}_{\mathrm{w}}-\mathrm{T}_{\mathrm{g}}\right)+\frac{\left(\mathrm{P}_{\mathrm{w}}-\mathrm{P}_{\mathrm{g}}\right) \mathrm{T}_{\mathrm{w}}}{\frac{\mathrm{M}_{\mathrm{a}} \mathrm{P}_{\mathrm{t}}}{\mathrm{M}_{\mathrm{a}}-\mathrm{M}_{\mathrm{w}}}-\mathrm{P}_{\mathrm{w}}}
\end{aligned}
$$


Where $h_{c}$ is free convective heat transfer coefficient, $R_{a c}$ is Modified Rayleigh number, $\mathrm{k}_{\mathrm{f}}$ is thermal conductivity of humid air, $\mathrm{d}$ is average vertical distance between bed and glass, $\rho$ is density of humid air, $g$ is gravity constant, $\beta$ is Expansion factor, $\mu$ is dynamic viscosity of humid air, $\alpha$ is thermal diffusivity of humid air, $\mathrm{P}_{\mathrm{w}}$ is saturation vapor pressure of water vapor at evaporation surface, $\mathrm{P}_{\mathrm{g}}$ is saturation vapor pressure of water vapor at condensation surface, $\mathrm{P}_{\mathrm{t}}$ is total pressure, $\mathrm{M}_{\mathrm{a}}$ is molecular weight of dry air and molecular weight of water vapor. Hence, the total energy transferred via convection is:

$$
\mathrm{E}_{\mathrm{c}}=\mathrm{t}_{\mathrm{cycle}} \mathrm{h}_{\mathrm{c}} \mathrm{A}_{\mathrm{b}}\left(\mathrm{T}_{\mathrm{w}}-\mathrm{T}_{\mathrm{g}}\right)
$$

Where, $A_{b}$ is adsorbent bed area and $t_{\text {cycle }}$ is desorption time.

The glass temperature for each desorption temperature was slightly different. For desorption temperatures 60,70 and $80{ }^{\circ} \mathrm{C}$, the average glass temperature was 33,34 and $35^{\circ} \mathrm{C}$, respectively. Figure S13 shows the energy consumption of the system tor various desorption temperature, which illustrates that the $70{ }^{\circ} \mathrm{C}$ is the best desorption temperature in terms of energy consumption. 


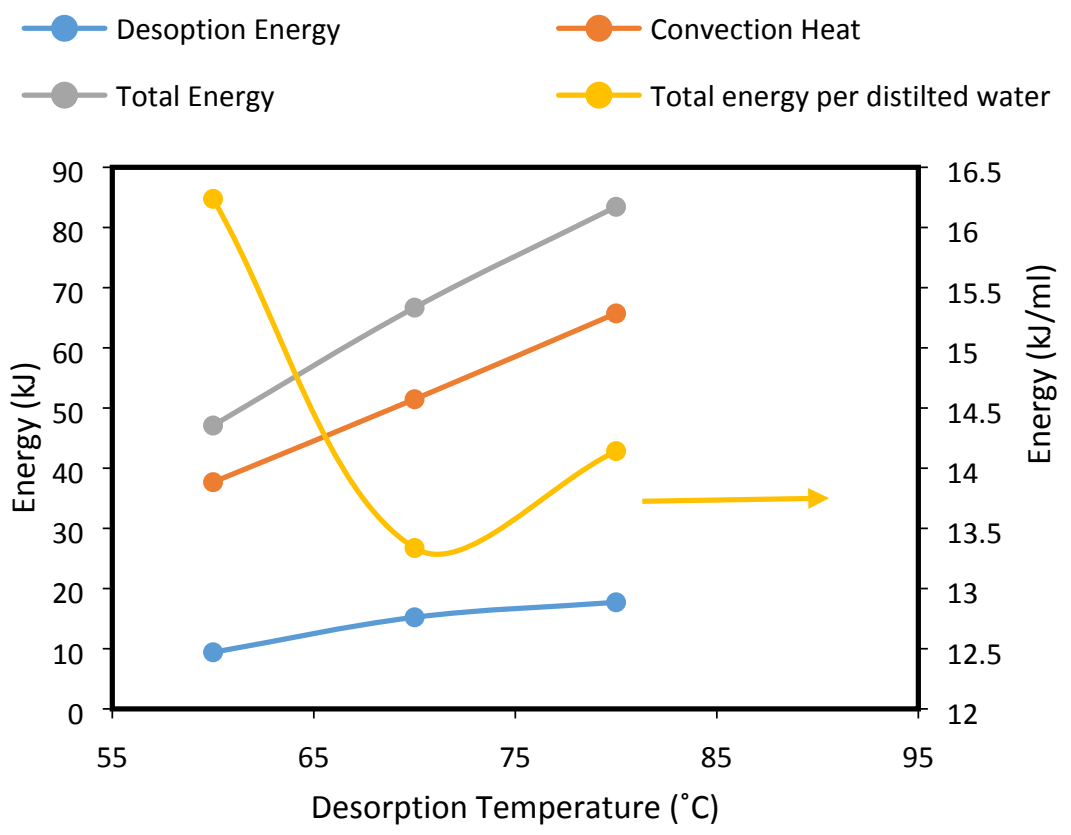

Figure S13. Energy consumption in the prototype for different desorption temperature.

\section{S12- Effect of desorption cycle length on the collected water amount}

In this study we considered one cycle per day and for desorption period we fixed at 7 hours. It might be higher water collection rate at the beginning of process in the case of $80^{\circ} \mathrm{C}$ but the whole amount of water in 7 hours was not so different. We observed that continuing heating in $60{ }^{\circ} \mathrm{C}$ can still evaporate the water in the bed at very slow rate but it must be considered that longer period leads to the lower water production per energy consumption. We repeated the test for $70{ }^{\circ} \mathrm{C}$ and $80^{\circ} \mathrm{C}$ desorption time in $1,2,5$, and 7 hours and the results are presented in Table S7. As can be seen, the amount of collected 
water in $80^{\circ} \mathrm{C}$ is more in shorter desorption time but as the desorption time prolongs the difference for collected water in $70^{\circ} \mathrm{C}$ and $80^{\circ} \mathrm{C}$ gets less.

Table S7. The test of different desorption time in two different desorption temperature in a bed containing $5 \mathrm{~g}$ Bina/FCNT which is left in the room overnight.

\begin{tabular}{|c|c|c|c|c|c|}
\hline Desorption temperature & & $1 \mathrm{hr}$ & $2 \mathrm{hr}$ & $5 \mathrm{hr}$ & $7 \mathrm{hr}$ \\
\hline \multirow{2}{*}{$70^{\circ} \mathrm{C}$} & Adsorbed water $(\mathrm{g})$ & 8.091 & 7.808 & 8.293 & 8.382 \\
\cline { 2 - 6 } & Collected water $(\mathrm{ml})$ & 0 & 1.1 & 3.8 & 4.7 \\
\hline \multirow{3}{*}{$80^{\circ} \mathrm{C}$} & Adsorbed water $(\mathrm{g})$ & 7.703 & 8.261 & 8.05 & 7.519 \\
\cline { 2 - 7 } & Collected water $(\mathrm{ml})$ & 2.2 & 3.7 & 4.5 & 4.8 \\
\hline
\end{tabular}

\section{S13-Thermal stability}

The thermal degradation behavior of the saturated hydrogel was investigated using the TGA method with a temperature rate increase of $5 \mathrm{~K} / \mathrm{min}$. Thermal stability improves with cyclic organic structure (aromatic cycles) in the polymer and crosslinking process [33]. However, the existence of double bonds or oxygen in the structure of polymers makes them more sensitive to high temperatures. The thermal decomposition of samples is a two-step process (Figure S14). The first step 
occurs at a temperature higher than $50^{\circ} \mathrm{C}$ and is related to water evaporation. The second degradation step starts at about $150^{\circ} \mathrm{C}$.

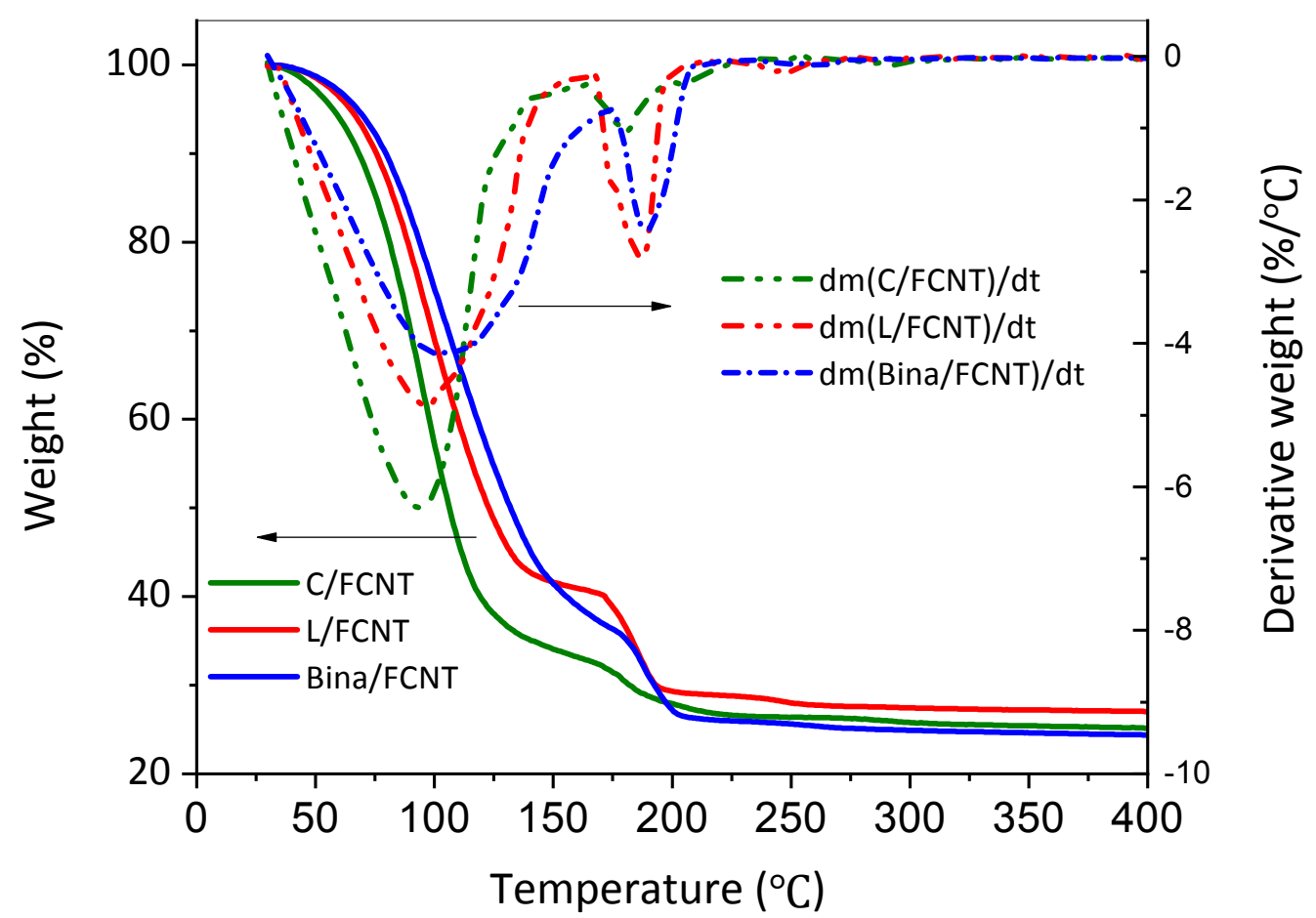

Figure S14. Thermal stability of Bina/FCNT, C/FCNT and L/FCNT in a baseline with temperature rise rate.

\section{S14- The composites' durability}

One of concerns in adsorbents is the delinquency phenomenon. Freeze-drying method or vacuum drying could help to keep the porosity of gel after water evaporation, but still the amount of adsorbed water is higher than sorbents volume. To see the durability in cycles, since the material is amorphous, XRD can not be so informative. One criterion can be the salt content before and after several 
cycles. To test the salt content in the sorbent, the sorbent beads before and after 20 cycles was subjected to ICP-OES (inductively coupled plasma optical emission spectrometry) to investigate the salt content. The concentration of ions in them is measured and the amount of salt in the composite is calculated. The salt content before the tests was $86 \%$, while after the cycles it did not alter ( $85 \%$ salt content). As can be seen in Figure S15, the beads kept their shape during 20 sorption process and the ion concentration in the sorbents (picked from the top layer) is almost constant.
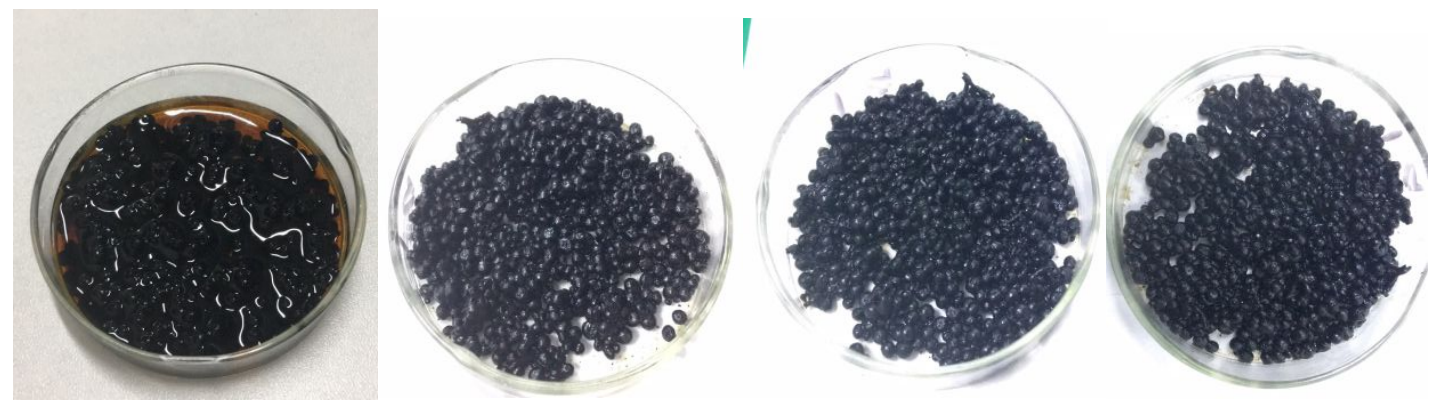

Figure S15. optical image of multi layer samples : a. immersed in adsorbed water (one night at ambient condition), b. 5 cycles, c. ten cycles. and d. 20 cycles. 


\section{Reference}

[1] X. Wang et al., "An Interfacial Solar Heating Assisted Liquid Sorbent Atmospheric Water Generator," Angewandte Chemie International Edition, vol. 58, no. 35, pp. 1205412058, 2019/08/26 2019.

[2] J. Y. Wang, J. Y. Liu, R. Z. Wang, and L. W. Wang, "Experimental investigation on two solar-driven sorption based devices to extract fresh water from atmosphere," Applied Thermal Engineering, vol. 127, pp. 1608-1616, 2017/12/25/2017.

[3] K. Matsumoto, N. Sakikawa, and T. Miyata, "Thermo-responsive gels that absorb moisture and ooze water," Nature Communications, vol. 9, no. 1, p. 2315, 2018/06/13 2018.

[4] L. Hou and P. Wu, "Exploring the hydrogen-bond structures in sodium alginate through two-dimensional correlation infrared spectroscopy," Carbohydrate polymers, vol. 205, pp. 420-426, 2019.

[5] A. A. Javidparvar, R. Naderi, and B. Ramezanzadeh, "Designing a potent anti-corrosion system based on graphene oxide nanosheets non-covalently modified with cerium/benzimidazole for selective delivery of corrosion inhibitors on steel in $\mathrm{NaCl}$ media," Journal of Molecular Liquids, vol. 284, pp. 415-430, 2019/06/15/ 2019.

[6] S. M. S. Basu, S. Senthil Kumar, B. Krishnamoorthy, S. Kumar Basu "Development and evaluation of calcium alginate beads prepared by sequential and simultaneous methods, , Brazilian Journal of Pharmaceutical Sciences,. 46 (2010) 765-790. http://www.scielo.br/pdf/bjps/v46n4/21.pdf (accessed June 30, 2019).".

[7] H. Daemi and M. Barikani, "Synthesis and characterization of calcium alginate nanoparticles, sodium homopolymannuronate salt and its calcium nanoparticles," Scientia Iranica, vol. 19, no. 6, pp. 2023-2028, 2012/12/01/2012.

[8] A. A. Javidparvar, B. Ramezanzadeh, and E. Ghasemi, "Effect of Various Spinel Ferrite Nanopigments Modified by Amino Propyl Trimethoxy Silane on the Corrosion Inhibition Properties of the Epoxy Nanocomposites," CORROSION, vol. 72, no. 6, pp. 761-774, 2016/06/01 2016.

[9] S.-Y. Jeon, J.-M. Yun, Y.-S. Lee, and H.-I. Kim, "Removal of Cu (II) ions by alginate/carbon nanotube/maghemite composite magnetic beads," Carbon Letters (Carbon Lett.), vol. 11, no. 2, pp. 117-121, 2010.

[10] D. Liu, W. Zhao, S. Liu, Q. Cen, and Q. Xue, "Comparative tribological and corrosion resistance properties of epoxy composite coatings reinforced with functionalized fullerene C60 and graphene," Surface and Coatings Technology, vol. 286, pp. 354-364, 2016/01/25/ 2016

[11] A. A. Javidparvar, R. Naderi, B. Ramezanzadeh, and G. Bahlakeh, "Graphene oxide as a $\mathrm{pH}$-sensitive carrier for targeted delivery of eco-friendly corrosion inhibitors in chloride solution: Experimental and theroretical investigations," Journal of Industrial and Engineering Chemistry, vol. 72, pp. 196-213, 2019/04/25/ 2019.

[12] H. Allaboun, M. M. Fares, and A. F. Abu Al-Rub, "Removal of Uranium and Associated Contaminants from Aqueous Solutions Using Functional Carbon Nanotubes-Sodium Alginate Conjugates," Minerals, vol. 6, no. 1, 2016.

[13] B. Joddar, E. Garcia, A. Casas, and C. M. Stewart, "Development of functionalized multi-walled carbon-nanotube-based alginate hydrogels for enabling biomimetic technologies," Scientific Reports, Article vol. 6, p. 32456, 08/31/online 2016. 
[14] Y. Li et al., "Removal of copper from aqueous solution by carbon nanotube/calcium alginate composites," Journal of Hazardous Materials, vol. 177, no. 1, pp. 876-880, 2010/05/15/2010.

[15] J. P. HOLMAN, Heat Transfer. 1968.

[16] X. Li, Y. Mo, W. Qing, S. Shao, C. Y. Tang, and J. Li, "Membrane-based technologies for lithium recovery from water lithium resources: A review," Journal of Membrane Science, vol. 591, p. 117317, 2019.

[17] Y. Wang, L. Zhang, J. Wu, M. N. Hedhili, and P. Wang, "A facile strategy for the fabrication of a bioinspired hydrophilic-superhydrophobic patterned surface for highly efficient fog-harvesting," Journal of Materials Chemistry A, 10.1039/C5TA04930J vol. 3, no. 37, pp. 18963-18969, 2015.

[18] H. Zhu, F. Yang, J. Li, and Z. Guo, "High-efficiency water collection on biomimetic material with superwettable patterns," Chemical Communications, 10.1039/C6CC05857D vol. 52, no. 84, pp. 12415-12417, 2016.

[19] P. Li, X. Hou, L. Qu, X. Dai, and C. Zhang, "PNIPAM-MAPOSS hybrid hydrogels with excellent swelling behavior and enhanced mechanical performance: preparation and drug release of 5-fluorouracil," Polymers, vol. 10, no. 2, p. 137, 2018.

[20] H.-T. Chou, Y.-C. Chen, C.-Y. Lee, H.-Y. Chang, and N.-H. Tai, "Biomimetic structure of carbon fiber cloth grafted with poly(N-isopropylacrylamide) for water collection and smart gates," RSC Advances, 10.1039/C7RA05869A vol. 7, no. 72, pp. 45799-45806, 2017.

[21] B. Wang, Y. Zhang, W. Liang, G. Wang, Z. Guo, and W. Liu, "A simple route to transform normal hydrophilic cloth into a superhydrophobic-superhydrophilic hybrid surface," Journal of Materials Chemistry A, 10.1039/C4TA00833B vol. 2, no. 21, pp. 7845-7852, 2014.

[22] A. J. Rieth, S. Yang, E. N. Wang, and M. Dincă, "Record Atmospheric Fresh Water Capture and Heat Transfer with a Material Operating at the Water Uptake Reversibility Limit," ACS Central Science, vol. 3, no. 6, pp. 668-672, 2017/06/28 2017.

[23] S. M. T. Abtab et al., "Reticular chemistry in action: a hydrolytically stable MOF capturing twice its weight in adsorbed water," Chem, vol. 4, no. 1, pp. 94-105, 2018.

[24] J. Y. Wang, R. Z. Wang, Y. D. Tu, and L. W. Wang, "Universal scalable sorption-based atmosphere water harvesting," Energy, vol. 165, pp. 387-395, 2018.

[25] P. A. Kallenberger and M. Fröba, "Water harvesting from air with a hygroscopic salt in a hydrogel-derived matrix," Communications Chemistry, vol. 1, no. 1, p. 28, 2018.

[26] R. Li, Y. Shi, M. Alsaedi, M. Wu, L. Shi, and P. Wang, "Hybrid Hydrogel with High Water Vapor Harvesting Capacity for Deployable Solar-Driven Atmospheric Water Generator," Environmental Science \& Technology, vol. 52, no. 19, pp. 11367-11377, 2018.

[27] P. A. Kallenberger, K. Posern, K. Linnow, F. J. Brieler, M. Steiger, and M. Fröba, "Alginate-Derived Salt/Polymer Composites for Thermochemical Heat Storage," Advanced Sustainable Systems, vol. 2, no. 7, p. 1700160, 2018.

[28] A. Permyakova et al., "Design of salt-metal organic framework composites for seasonal heat storage applications," Journal of Materials Chemistry A, 10.1039/C7TA03069J vol. 5, no. 25, pp. 12889-12898, 2017. 
[29] H. Furukawa et al., "Water Adsorption in Porous Metal-Organic Frameworks and Related Materials," Journal of the American Chemical Society, vol. 136, no. 11, pp. 4369-4381, 2014/03/19 2014.

[30] L. Garzón-Tovar, J. Pérez-Carvajal, I. Imaz, and D. Maspoch, "Composite Salt in Porous Metal-Organic Frameworks for Adsorption Heat Transformation," Advanced Functional Materials, vol. 27, no. 21, p. 1606424, 2017.

[31] F. Zhao, X. Zhou, Y. Liu, Y. Shi, Y. Dai, and G. Yu, "Super Moisture-Absorbent Gels for All-Weather Atmospheric Water Harvesting," Advanced Materials, vol. 31, no. 10, p. 1806446, 2019.

[32] H. Zheng, X. Zhang, J. Zhang, and Y. Wu, "A group of improved heat and mass transfer correlations in solar stills," Energy Conversion and Management, Article vol. 43, no. 18, pp. 2469-2478, 2002.

[33] K. Król-Morkisz and K. Pielichowska, "13 - Thermal Decomposition of Polymer Nanocomposites With Functionalized Nanoparticles," in Polymer Composites with Functionalized Nanoparticles, K. Pielichowski and T. M. Majka, Eds.: Elsevier, 2019, pp. 405-435. 